\title{
Twitter Brand-Directors: el efecto marca en las redes sociales de los directores de medios españoles
}

\author{
Víctor HERRERO-SOLANA \\ Universidad de Granada \\ victorhs@ugr.es \\ Magdalena TRILlo-DoMíNGUEZ \\ Universidad de Granada \\ mtrillo@ugr.es /mtrillo@granadahoy.com
}

Recibido: 8 de octubre de 2013

Aceptado: 10 de marzo de 2014

\section{Resumen}

Contexto. Twitter es una de las herramientas más utilizadas hoy en día por los periodistas de todo el mundo. Una de las medidas de visibilidad más importantes de una cuenta de Twitter es la cantidad de seguidores. En el presente trabajo se analiza el comportamiento de los medios de comunicación españoles y de sus directores, a la luz de este indicador. Material y métodos. Se utilizan los seguidores de las cuentas de Twitter tanto de los medios como de sus directores. Con los datos se realizan dos rankings y una representación bidimensional. Resultados y conclusiones. Encontramos un grupo de directores que son su propia marca (brand-directors): Ignacio Escolar, Pedro J. Ramírez, Julio Alonso y Alfredo Relaño, entre otros. También encontramos medios con mucha visibilidad en Twitter pero con directores que no la tienen: El País, Muy Interesante, Marca, RTVE, entre otros.

Palabras clave: Periodismo, redes sociales, Twitter, liderazgo, prensa, liderazgo, director-marca

\section{Twitter Brand-Directors: \\ the brand effect of spanish media directors on social networks}

\begin{abstract}
Background. Twitter is one of the tools used today by journalists from around the world. One measure of visibility of a Twitter account is the number of followers. In this paper we analyze the behavior on Twitter in the Spanish media and its directors. Material and methods. We use the followers of the directors and media Twitter accounts. With this data we make two rankings and a bidimensional chart. Results and conclusions. We found a group of directors who are their own brand (brand-directors): Ignacio Escolar, Peter J. Ramirez, Julio Alonso and Alfredo Relaño, among others. We also found medi with high visibility in Twitter but the directors do not: El País, Muy Interesante, Marca, RTVE, among others.

Keywords: Journalism, Social Media, Twitter, leadership, press, brand-director

\section{Referencia normalizada}

HERRERO-SOLANA, Víctor y TRILLO-DOMÍNGUEZ, Magdalena (2014): “Twitter Brand-Directors: el efecto marca en las redes sociales de los directores de medios españoles". Estudios sobre el Mensaje Periodístico. Vol. 20, Núm. 1 (enero-junio), págs.: 131-146. Madrid, Servicio de Publicaciones de la Universidad Complutense.
\end{abstract}

Sumario: 1. Introducción. 2. Material y métodos. 3. Resultados y discusión. 4. Conclusiones. 5. Referencias bibliográficas.

\section{Introducción}

En el escenario global de profundo deterioro que está viviendo el periodismo, tanto desde el punto de vista económico por el impacto de la crisis como en lo referente a la transformación que están imponiendo las nuevas tecnologías en el ecosistema de 
medios y a la creciente crisis de credibilidad que sufre todo el sector, las redes sociales están ocupado un espacio cada vez más importante en las rutinas de los profesionales de la información y en las estrategias de comunicación: cumplen un papel de vigilancia respecto a lo que se publica y difunde en los medios tradicionales, se han sumado en el quehacer periodístico como fuentes informativas de primer nivel, son un altavoz imprescindible para mover y proyectar los contenidos y son un termómetro para detectar los puntos calientes de la actualidad informativa.

Si nos circunscribimos al sector prensa y a la red social Twitter como objeto específico de este trabajo, podríamos llamar la atención sobre los cambios de tendencias que se están observando en la difícil relación y creciente competencia que el auge y expansión de internet ha significado para los medios impresos desde los años 90. Aunque aún hoy el modelo digital de los cibermedios sigue sin ser rentable, las redes sociales están desarrollando un ecosistema informativo propio donde se ha pasado de la competencia a la colaboración y de la redundancia a la asunción de un papel de producción e intercambio de información que termina repercutiendo de forma positiva en los medios impresos por la enorme visibilidad que implica.

En este contexto, el tradicional esquema de la comunicación se rompe y los periodistas se ven obligados a "aprender" un nuevo papel donde los productores y consumidores de información son intercambiables, los filtros y controles son inmediatos $\mathrm{y}$ tremendamente exigentes $\mathrm{y}$, al mismo tiempo, desaparecen los principios y rutinas que tradicionalmente han diferenciado el trabajo periodístico y que han generado credibilidad tanto en lo referente a las fuentes como a los propios contenidos.

Es evidente que estamos ante nuevo ecosistema de medios y ante un nuevo paradigma en cuanto a modos de producción, difusión y consumo de información en el que podríamos recuperar el debate sobre si la prensa estaría llamada a desaparecer. Por un lado, podríamos invocar los insistentes vaticinios sobre la "muerte del papel" y poner sobre la mesa los datos sobre la caída de venta de ejemplares, desplome de la publicidad, cierre de cabeceras y hundimiento de ingresos en las empresas periodísticas con el consecuente adelgazamiento de plantillas vía reducciones y despidos- para ilustrar la radiografía más negra de la prensa de los dos últimos siglos, el momento más crítico desde su revulsiva expansión en el siglo XIX como un verdadero medio de masas.

Pero, muy al contrario, con este trabajo queremos reivindicar el papel de referencia y de liderazgo que los periódicos, dinosaurios de la información, están desempeñando en la incorporación, convivencia y readaptación de los medios en el escenario digital. Hablamos del "efecto marca" (brand effect) y de cómo en el desarrollo de las redes sociales es la prensa, un vez más, la que está determinando las dinámicas, la que está definiendo el presente y el futuro del sector. Lo veremos detalladamente al profundizar en la investigación que nos ocupa tanto en el caso de la visibilidad y posicionamiento del propio medio en Twitter como desde una perspectiva nueva que queremos introducir sobre el papel concreto que desempeñan los directores en la red de microblogging con la creciente apuesta por dar el salto a la web 2.0 como una forma de proyectar su marca personal. 
Antes de pasar a desarrollar la investigación propiamente dicha, debemos subrayar que se inserta en la misma línea de trabajo que ya comenzamos en 2008 con la realización de la tesis doctoral Análisis cibermétrico de la prensa digital española: ranking de calidad web y mapa de influencia mediática (Trillo-Domínguez, 2008) y el CAMEO de Marshall McLuhan (Trillo-Domínguez et al., 2008) en cuanto a las estrategias metodológicas empleadas y el esfuerzo por continuar el la utilización de técnicas del ámbito de la Documentación a la investigación en Comunicación, especialmente las que tengan que ver con la recuperación y visualización de la información (Herrero-Solana, 2005: web).

Desde el punto de vista del contenido y de la idea de marca (brand) que hemos apuntado, nos gustaría destacar que ya en el trabajo sobre la prensa digital se observaba cómo dicha marca y la propia credibilidad que aún hoy acompaña a la prensa escrita respaldaban su posición en el ciberespacio según los diferentes rankings y el mapa de influencia elaborados y la situaban en una posición privilegiada. La idea de marca se trasladaba a la red, de modo que era las ediciones digitales de algunos de los periódicos de referencia en el marco de la prensa española los que lideraban las diferentes clasificaciones llevadas a cabo.

Como ya apuntaba la profesora Concepción Edo en su libro Del papel a la pantalla, "se ha demostrado que el prestigio de la marca de un diario que vende cientos de miles de ejemplares en papel pesa mucho a la hora de informarse en Internet", algo que se puede corroborar al analizar las audiencias en prensa y en digital de medios como la CNN, The Washington Post, USA Today y, en España, El País, El Mundo o La Vanguardia (Edo Bolós, 2002).

Para la docente, las soluciones de futuro de las empresas periodísticas están directamente relacionadas con la actualización constante de la información, la fidelidad a la imagen de marca, la utilización de vídeo y audio mediante alianzas con otros medios, una mayor interacción con el usuario y la transformación de las secciones en centros de coordinación y sindicación de contenidos. Y recordaba, además, que ni siquiera la insistente muerte del papel es el debate más importante, ya que la letra impresa no va a desaparecer aunque cambie de soporte y, sobre todo, "porque al menos durante bastantes años tampoco va a sucumbir el papel-prensa que, probablemente, se convertirá en garantía de análisis plural y de credibilidad ante el aluvión informativo que ofrecen tantos portales nacionales e internacionales, cadenas de televisión, emisoras de radio, periódicos, revistas, empresas o páginas individuales presentes en la red".

Compartimos su reflexión y consideramos que el estudio que ahora presentamos viene a corroborarla: en internet, en las redes sociales, hay sobreabundancia de información pero no toda genera credibilidad y confianza en los lectores y usuarios. Una situación que termina dando ventaja a los diarios con una amplia trayectoria, influencia y prestigio por su publicación en papel y, como veremos en el trabajo, a los máximos responsables de tales medios; en unos casos con un impacto propio y personal de marca y en otros 'arrastrados' por la posición de su medio.

Siguiendo al profesor Salaverría, podríamos hablar en este punto de las necesarias sinergias y de la imprescindible "convergencia" que para los diarios ha supuesto el salto digital: "Los diarios se aproximan al cambio más importante de su historia: el 
momento en que el papel, arrinconado por nuevas formas de consumo informativo de una nueva generación de lectores, deberá dejar paso al soporte digital. Y ya es seguro que ocurrirá; la única duda es cuándo" (Salaverría, 2007: 4).

En concreto, el estudio lo hemos querido centrar en Twitter por considerar que se trata de la plataforma en línea que mayor impacto ha tenido sobre los medios y el periodismo. Como enfatiza el profesor Orihuela en 80 claves sobre el futuro del periodismo, "todas las grandes catástrofes naturales, atentados terroristas, accidentes, procesos electorales, movimientos sociales y acontecimientos deportivos han encontrado en Twitter el espacio natural para despegar como breaking news. Los periodistas utilizan Twitter para buscar y monitorizar fuentes, detectar noticias y tendencias, y los medios para promocionar sus contenidos y, en el mejor de lo casos, para dialogar con sus usuarios. Hoy, así como prácticamente no hay medio en línea que no disponga de una buena oferta de blogs, tampoco hay ya medios relevantes que no tengan una presencia destacada en Twitter" (Orihuela, 2012). Los ejemplos y las apuestas son múltiples. Orihuela destaca, por ejemplo, la apuesta por la "Tuitosfera" y el microblogging como plataforma de difusión y conversación, como formato para coberturas en tiempo real e, incluso, como un modo de acercar los procesos internos de la redacción a los usuarios.

A nuestro juicio, ésta es una cuestión esencial que podremos ver al analizar la actividad de los directores de medios españoles: si se está innovando realmente en los modos de comunicación periodística, si se trata de una presencia meramente testimonial, si lo que se busca es crear influencia. Intercambiar 140 caracteres en la red empieza a ser un factor más que sumar a las estrategias de "subsistencia" de los medios, mucho más importante que "una moda pasajera" como dijo hace un par de años Nicholas Negroponte subestimando una creciente realidad de dinámicas de comunicación que, como bien subraya Orihuela, supone ya "un modo social de leer la realidad y de leer los medios en tiempo real y a escala global".

Bien es cierto que todavía pesa más esa utilización de la Tuitosfera como altavoz que como un espacio de conversación y escucha real y, menos aún, como una extensión natural del espacio de los medios para la comunicación e interactuación con lectores y usuarios. El estudio que Tristán Elósegui ha realizado al respecto es bastante clarificador. El experto en marketing online recurre a la ratio seguidos/seguidores y al índice de influencia en medios sociales Klout para constatar que "existe un gran desequilibrio entre la cantidad de seguidores que tienen la cuentas de los medios y la cantidad de usuarios a los que siguen", al mismo tiempo que se observa "manifiestamente mejorable la calidad de la interacción los usuarios". En "Los medios usan Twitter como un altavoz" (Elósegui, 2011: web) analiza el uso de la red en 27 de los medios españoles más importantes (prensa, radio, televisión y cibermedios) y concluye cómo se utiliza Twitter para difundir sus contenidos y cómo apenas se escucha y responde a los usuarios.

Sin hablar directamente de la marca del medio, realiza una observación estrechamente ligada con nuestra investigación: "A pesar de los datos algunos de los medios estudiados siguen siendo influyentes en Twitter porque su influencia offline aún es muy fuerte y los seguimos de forma automática, por costumbre, los usamos puramente como fuente de información, no esperamos más de ellos". Un potencial que, como 
también veremos en nuestro trabajo, están desaprovechando los medios, especialmente, los que ya tienen una posición de prestigio y liderazgo fuera de las redes que los sitúan en una situación de clara ventaja para competir. En el estudio de Elósegui el medio que mejor se posiciona es precisamente El País y es curioso que, dentro de sus conclusiones, también recalque cómo "la prensa es mucho más influyente que la radio y la televisión" confirmando esa dinámica de liderazgo que ya hemos mencionado en cuanto al papel de innovación del sector prensa.

Desde la perspectiva general del uso de las redes sociales en la profesión, es especialmente revelador el estudio realizado por el Laboratorio de Investigación Periodística de la Universidad Carlos III (Labàpart) sobre Twitter. En él se recoge cómo el 90\% de los periodistas encuestados consideran Twitter como la mayor red social para buscar información y contactar con fuentes y cómo el periodista dedica cada vez más tiempo a navegar por la Red con fines profesionales (Carrera-Álvarez et al., 2012: 42).

El estudio viene a demostrar la utilidad efectiva que tiene Twitter para los periodistas españoles como herramienta para investigar y completar las estrategias de trabajo tradicionales, del mismo modo que ya lo constató en 2010 Ali Nobil Ahmad analizando The Guardian y las coberturas realizadas en acontecimientos como el G20 (Nobil Ahmad, 2010: 145): ¿puede ser Twitter una oportunidad de negocio para los medios? ¿un revulsivo para fomentar el periodismo crítico e innovador? ¿puede convertirse el periodismo en una "herramienta útil" para Twitter?

Como ya hemos indicado, en el caso del presente trabajo nuestro interés se centra en la presencia en Twitter de los directores de medios españoles, y con ese fin nos planteamos tres preguntas que nos puedan servir como guías de su desarrollo. Ellas son:

¿Constituye la cuenta de Twitter de un medio una plataforma sobre la que se proyectan las cuentas de sus directores?

¿Existen directores con cuentas de Twitter más seguidas que la de sus propios medios?

¿Cuál es, en términos generales, la ratio de seguidores de las cuentas de los directores vs. los seguidores de las cuentas de los medios?

\section{Material y métodos}

Para identificar las cuentas de Twitter recurrimos a la lista de directores de medios de comunicación de España que creó el profesor Ramón Salaverría a partir de 2011 y que se sigue viva y actualizándose en la propia red social.

Según la información facilitada por el propio investigador, profesor titular de Periodismo y director del Departamento de Proyectos Periodísticos de la Universidad de Navarra, la primera versión de la lista se publicó el 2 de marzo de 2012 con medio centenar de periodistas. A partir de ahí, gracias a sus propias indagaciones y a aportaciones externas, se he ido ampliando con más de un centenar de medios ${ }^{1}$. En este tiempo,

${ }^{1}$ La consulta realizada para la recuperación de datos, a finales de junio de 2013, registraba 116 miembros. En julio ya llegaba a los 121: https://twitter.com/i/\#!//rsalaverria/directoresmedios 
también ha dado de baja a algunos usuarios, porque han sido relevados en sus anteriores responsabilidades directivas o bien porque el medio ha desaparecido.

Para hallar las cuentas de los usuarios, el profesor Salaverría ha recurrido a varias estrategias: localización a través de retuits por parte de usuarios en Twitter; búsquedas avanzadas con Google y mediante el propio buscador de Twitter; y sugerencias recibidas por parte de usuarios de la red social.

Respecto a los criterios para la inclusión en la lista, que los propios profesionales e investigadores del sector han tomado como un referente sobre el salto dado por los directivos de medios a las redes sociales y en concreto a la utilización de Twitter en sus rutinas de trabajo, el listado incluye tanto directores como directores adjuntos o subdirectores de cualquier tipo de medio (prensa, radio, televisión e internet), y a cualquier escala (nacional, regional y local). En el caso de los medios audiovisuales no se ha incluido a los directores de programas (perfiles especialmente mediáticos como los de Pepa Bueno, Carlos Herrera, Sáenz de Buruaga o similares) ni tampoco a los directores comerciales o directores técnicos. En los dos casos supondría una distorsión respecto al perfil estrictamente periodístico que caracteriza el listado.

Desde un punto de vista periodístico, podríamos cuestionar la pertinencia de mantener en listado a destacados blogueros y activistas de las redes como Julio Alonso porque, más allá de que sean directores de una empresa del ámbito de la comunicación, no responden al concepto clásico de director de medio. No obstante, hemos optado por mantener el listado original elaborado por el equipo de Salaverría y realizar las observaciones oportunas a la hora de analizar la posición de los diferentes medios.

En primer lugar, antes de analizar los indicadores y gráficos propiamente dichos, es importante hacer una valoración de la lista. En ella podemos observar ya ciertas dinámicas respecto a la apuesta que los directivos de los medios de comunicación españoles están realizando no sólo por estar presentes en la red social de mayor expansión en el campo periodístico sino también por lograr influencia en cuanto a seguidores y visibilidad en los debates públicos de actualidad.

Centrándonos en el sector prensa, el que tradicionalmente ha marcado (y sufrido) las nuevas estrategias de comunicación derivadas de la incorporación de las nuevas tecnologías y formas de trabajo, una primera conclusión a la que podemos llegar es que estamos en los albores de la incorporación real de Twitter como sistema de difusión y comunicación con los lectores por parte de los directivos de medios españoles. Aparentemente, como se ha saludado en el sector, "casi todos los directores de periódicos españoles" tienen cuenta en Twitter, tal y como se destaca en 233 grados, el popular blog sobre periodismo y medios de Lainformacion.com (Mark de Zabaleta, 2011).

Finalmente el conjunto total fue de 116 directores. Para cada uno de ellos se hizo una consulta manual en twitter.com durante la primer semana del mes de junio de 2013, con el fin de extraer los siguientes datos (siempre que estuvieran disponibles): nombre de cuenta, medio, función, cantidad de tuits, cantidad de seguidos, cantidad de seguidores. La misma tarea se llevó adelante con las cuentas de sus respectivos medios. El principal dato para el análisis es la cantidad de seguidores de cada cuenta, variable que ya fue utilizada en un estudio reciente sobre cuentas de Twitter de periodistas españoles, aunque los autores no han puesto tanto interés en la medición de 
los seguidores como en el análisis de los propios tuits (Rodríguez Ruibal, 2013: 965). Durante la recolección de datos detectamos que para las cuentas pequeñas y medianas este dato podía estar influido por el hecho de que se siguieran a su vez gran cantidad de usuarios. Para evitar este factor de quid pro quo decidimos trabajar con la diferencia entre el número de seguidores y el de cuentas seguidas. En término generales esto afectó poco a los principales actores del estudio. Esta información finalmente es presentada en forma de tabla de rankings y un gráfico bivariante. La totalidad de los datos utilizados en este trabajo se ha puesto en abierto y pueden ser consultados en la plataforma de open data Zenodo (Herrero-Solana, 2013: web).

\section{Resultados y discusión}

En la Tabla I vemos el ranking de directores con más seguidores. Como indicamos en el apartado anterior calculamos la diferencia entre seguidores y seguidos. Este efecto de corrección afecta muy poco a los elementos de la tabla ya que en todos los casos el volumen de seguidores es muy superior al de seguidos (entre 20 y 100 veces mayor). A su lado podemos ver una tabla similar con la información de las cuentas de Twitter de los propios medios. En este caso la diferencia entre seguidores y seguidos es aún mayor.

Si observamos los primeros puestos del ranking de directores, se revela ya la situación de liderazgo que ocupan periodistas con una larga trayectoria y una intensa actividad en las redes sociales como son Ignacio Escolar y Julio Alonso y dos de los directores más mediáticos de la prensa actual: Pedro J. Ramírez y Alfredo Relaño.

Tabla I - Directores

\begin{tabular}{|rlc|}
\hline \# & Director & $\begin{array}{c}\text { seguidores } \\
\text { - seguidos }\end{array}$ \\
\hline $\mathbf{1}$ & Ignacio Escolar (Eldiario.es) & 255635 \\
\hline $\mathbf{2}$ & Pedro J. Ramírez (El Mundo) & 171018 \\
\hline $\mathbf{3}$ & Julio Alonso (Weblogs) & 117036 \\
\hline $\mathbf{4}$ & Alfredo Relaño (As) & 98420 \\
\hline $\mathbf{5}$ & Jesús Maraña (Público) & 59315 \\
\hline $\mathbf{6}$ & Carles Capdevila (Ara.cat) & 51595 \\
\hline $\mathbf{7}$ & Arsenio Escolar (20 Minutos) & 48631 \\
\hline $\mathbf{8}$ & Montserrat Domínguez (Huffington) & 41800 \\
\hline $\mathbf{9}$ & Francisco Marhuenda (La Razón) & 38024 \\
\hline $\mathbf{1 0}$ & Gumersindo Lafuente (Huffington) & 36350 \\
\hline $\mathbf{1 1}$ & Javier Moreno (El País) & 34214 \\
\hline $\mathbf{1 2}$ & Juan Luis Sánchez (Eldiario.es) & 34073 \\
\hline $\mathbf{1 3}$ & Felipe del Campo (Marca TV) & 30760 \\
\hline $\mathbf{1 4}$ & Manuel Moreno (Trecebits) & 24092 \\
\hline $\mathbf{1 5}$ & Enric Juliana Ricart (La Vanguardia) & 20744 \\
\hline $\mathbf{1 6}$ & Virginia P. Alonso (20 Minutos) & 18808 \\
\hline $\mathbf{1 7}$ & Carmela Ríos (Terra) & 18578 \\
\hline $\mathbf{1 8}$ & Carolina Denia (Clipset) & 17688 \\
\hline $\mathbf{1 9}$ & Carlos Marañón (Cinemanía) & 13825 \\
\hline $\mathbf{2 0}$ & Antonio H. Rodicio (Cadena Ser) & 13642 \\
\hline & & \\
\hline
\end{tabular}

Tabla II - Medios

\begin{tabular}{|c|c|c|}
\hline & Medio & $\begin{array}{l}\text { seguidores } \\
\text { - seguidos }\end{array}$ \\
\hline 1 & muyinteresante & 2.744 .167 \\
\hline 2 & el_pais & 2.660 .894 \\
\hline 3 & marca & 1.374 .639 \\
\hline 4 & elmundoes & 1.124 .283 \\
\hline 5 & diarioas & 616.153 \\
\hline 6 & rtve & 557.867 \\
\hline 7 & $20 \mathrm{~m}$ & 351.070 \\
\hline 8 & abc_es & 345.402 \\
\hline 9 & publico_es & 336.942 \\
\hline 10 & EFEnoticias & 330.960 \\
\hline 11 & marieclaire_es & 296.255 \\
\hline 12 & telecincoes & 289.510 \\
\hline 13 & La_SER & 262.087 \\
\hline 14 & lasextaTV & 243.964 \\
\hline 15 & terraNoticias & 218.221 \\
\hline 16 & lavanguardia & 191.059 \\
\hline 17 & elconfidencial & 158.883 \\
\hline 18 & 24horasNoticias & 143.762 \\
\hline 19 & Expansión.com & 134.067 \\
\hline 20 & eldiarioes & 132.730 \\
\hline
\end{tabular}

En el caso de Ignacio Escolar, director de Diario.es, un gran activista de las redes sociales, se trata del periodista que más seguidores tiene de todo el estudio (255.635 en el momento de realizar la consulta), muy por encima del segundo, Pedro J. Ramí- 
rez director de El Mundo, (171.018) y Julio Alonso, al frente de Weblogs (117.036) y a bastante distancia de Relaño, director del diario As (98.420). Sin embargo esta situación no era así hace solo seis meses. Por entonces Escolar y Ramírez tenían una cantidad de seguidores más cercana, por sobre los 150.000. En solo seis meses, si bien ambos han crecido, Ignacio Escolar lo ha hecho en nada menos que casi 100.000 seguidores. Estos datos forman parte de un estudio dinámico que mostrará la evolución a lo largo de todo un año, que se encuentra aún en curso y que esperemos pueda ser publicado en los próximos meses.

Si bien con ambas tablas es posible apreciar la relación entre las cuentas de los directores y sus medios, creemos que es en la Figura 1 donde mejor se identifica este fenómeno. En el eje horizontal tenemos la cantidad de seguidores que tiene un determinado medio y en el eje vertical la cantidad de seguidores de su respectivo director. De esta forma logramos una ordenación espacial que facilita su análisis visual. Vemos que en términos generales los valores del eje horizontal son unas 10 veces mayores que los del vertical, ya que esta suele ser la relación de tamaño entre las cuentas de un medio y su director. La gran mayoría de casos aparecen abajo a la izquierda debido a que la mayoría de los directores y sus medios tienen una cantidad moderada/baja de seguidores, dentro de un rectángulo de algo menos de 50.000 vertical y algo más de 300.000 horizontal. Los casos que se encuentran fuera de este rectángulo son los verdaderamente destacables y por tanto los hemos etiquetado e identificado con cuadrados en lugar de rombos. Los puntos que predominan en el eje horizontal han sido etiquetados con el nombre del medio y los que destacan en el eje vertical con el nombre del director.

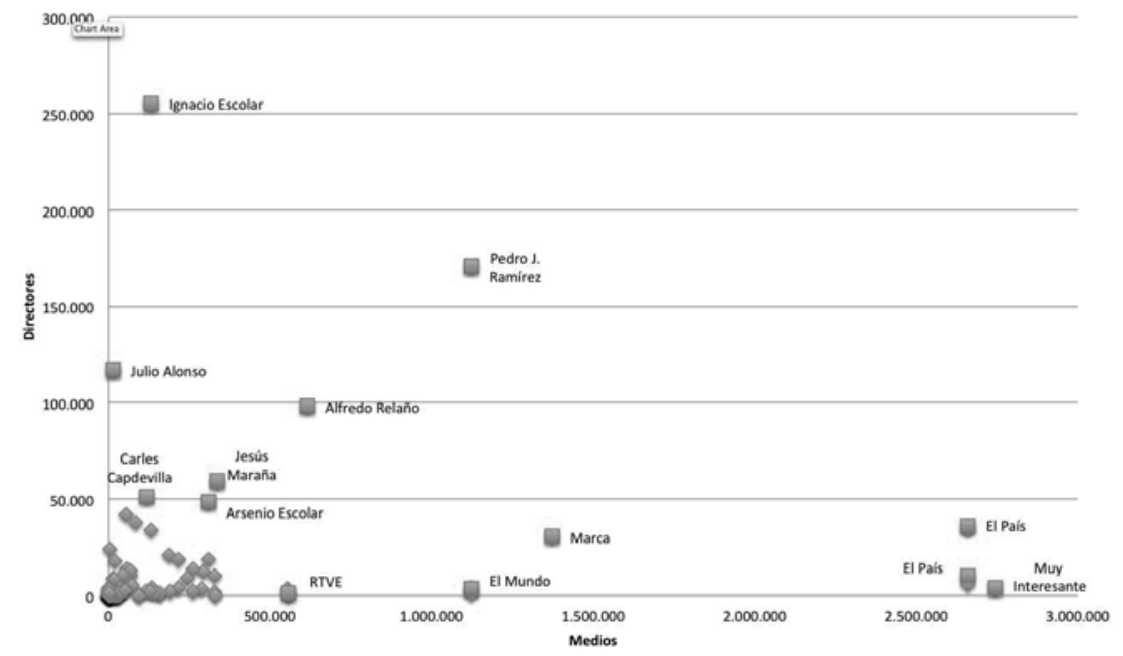

Figura 1 - Relación directores/medios según seguidores en Twitter

En esta primera aproximación se observa ya un clara diferenciación entre los directores que tienen detrás el impulso del efecto marca de su medio y de quienes se han construido su posicionamiento en Twitter superando la posición de sus medios de re- 
ferencia o incluso sin la existencia de un medio periodístico detrás como tal. Nos referimos a Ignacio Escolar y Julio Alonso: situados muy a la izquierda y en la parte superior del gráfico, se evidencia un liderazgo personal y se observa claramente cómo sus respectivos medios no compiten con su posicionamiento. Tenemos que tener en cuenta que Diario.es tiene una vida aún corta (nació en septiembre de 2012) por lo que podría ser interesante realizar un seguimiento para comprobar si se produce un despegue importante del ciberdiario y se comporta como las cabeceras con versión papel y marca consolidada o si, por el contrario, se mantiene el liderazgo de Escolar y en un segundo plano, el medio. De entrada, en apenas ocho meses ya ha conseguido situarse en el 'top 20' de medios que hemos realizado junto a algunos de los periódicos más potentes y con una apuesta más consolidada por la red. En este caso, y pese al normal movimiento de retroalimentación que se produce entre director y medio, podríamos inferir que es la propia posición de Escolar y su enorme proyección mediática la que está tirando del ciberdiario.

Ignacio Escolar ${ }^{2}$, con una amplísima trayectoria periodística que le ha llevado de diarios como Cinco Días y El Mundo a colaborar con revistas como Muy Interesante, Rolling Stone, Geo o Quo, fue uno de los primeros periodista que apostaron por internet de una forma profesional (dirigió El Navegante en Telecinco) y que han sabido hacerse un hueco como experto y consultor construyéndose una marca propia en las redes sociales como un gran generador de opinión. Su actividad como tertuliano en programas de radio y televisión y su proyección como director fundador del ya extinguido Público (de septiembre de 2007 hasta su destitución en enero de 2009, aunque siguió como columnista hasta la desaparición del periódico en marzo de 2012) han sido factores determinantes para explicar su influencia y su posicionamiento en Twitter. La fundación del diario digital Diario.es, con un planteamiento de gestión y micromecenazgo innovador en el panorama mediático español y unas prácticas éticas y de transparencia ejemplares, lo lleva a desvincularse de Publico.es (el periódico sigue en la red y también es uno de los diarios mejor posicionados según se observa en el ranking de medios) para buscar un espacio propio en el entorno digital. Aquí claramente el efecto de marca es el del propio director que logra doblar el número de seguidores que tiene su propio medio (relación 2:1 aproximadamente).

En lo referente a Julio Alonso, debemos recordar que es el fundador y director de Weblogs S.L., líder en publicaciones temáticas online en español, y que su campo de trabajo es precisamente los medios, internet y el activismo digital. Fue además en 2005, un año antes incluso del nacimiento de Twitter, cuando decidió dejar la consultoría Cluster y dedicarse a los blogs con la empresa que ya es líder en weblogs en español y la mayor de Europa. Su experiencia y trayectoria en el mundo de las redes es, por tanto, de las más sólidas y solventes de entre los directivos incluidos en la mues-

2 Colabora como articulista con El Periódico, es analista político de Las Mañanas de Cuatro y el Gran Debate de Telecinco, participa en la tertulia política de Hoy por Hoy de la Ser, colabora con RTVE, con Antena 3 y con La Sexta, etc. Es también socio fundador de Bitban, una consultora de tecnología y diseño para los medios de comunicación para internet, novelista y ensayista... 
tra si bien podríamos matizar si una persona de su perfil debería formar parte o no de este listado de directores de medios en Twitter. De hecho su ratio con el medio es mayor que la de Escolar y con más de 8:1.

Alonso ha destacado precisamente en más de una entrevista la "pasión" que para él supone el mundo del blog y ha recalcado la situación de desventaja que sufren los medios tradicionales para hacerse un hueco en un contexto dominado por aficionados con una enorme especialización temática y con una elevada credibilidad; algo que él ha sabido contrarrestar perfectamente. Al igual que en el caso de Escolar, el también responsable de Merodeando es uno de los principales prescriptores de tendencias y estrategias en el contexto digital y una de las voces más influyentes. Son líderes de opinión, por encima de sus medios. Una marcada situación de influencia nacida de la propia marca personal que les ha reportado su trayectoria.

Pedro J. Ramírez, posicionado en la parte superior justo detrás de Nacho Escolar pero desplazado hacia el centro, es el director de periódico que mejor refleja el equilibro, identificación y simbiosis que se establece entre su marca personal y la de su medio. Hablar de Pedro J. Ramírez es hablar de El Mundo; un medio del que ha sido el referente desde su fundación. Pedro J. Ramírez es uno de los directores más mediáticos del panorama periodístico español, uno de los más polemistas y más influyente. La intensidad de su actividad en Twitter es, además, la más elevada que se observa en toda la muestra, tanto por el tiempo que lleva en la red (fue uno de los primeros periodistas que apostaron por Twitter) como por la intensidad de su actividad tuitera (42.234 tuits en el momento de recoger la información). A bastante distancia de Ignacio Escolar (29.944 tuits), triplicando a Julio Alonso (13.146) (estos valores serán analizados en detalle en un posterior estudio de carácter dinámico ya mencionado).

En el caso de Pedro J., es El Mundo, con más de 1,1 millones seguidores (una relación 1:6) el que lo posiciona a la parte central del gráfico pero sin imponerse o absorber su protagonismo como ocurre en el caso de El País. Esto si ocurre con otros directivos del El Mundo (directores adjuntos) que se precipitan directamente hacia el eje horizontal. Por su parte, el diario del grupo Prisa, con más de 2,6 millones de seguidores, deja a su director, Javier Moreno, en una posición de insignificancia (43.495 seguidores), a mucha distancia de su competidor directo en los quioscos, en internet y en las redes sociales. En esta ausencia de liderazgo también hay que tener en cuenta el factor de su actividad en la red, ya que apenas contabiliza 1.600 tuits.

Desde el punto de vista de los medios ubicados abajo a la derecha, el posicionamiento que ocupa El País es de claro liderazgo como medio junto a la revista Muy Interesante. El caso de la conocida revista de divulgación requeriría una mención especial, ya que con cerca de tres millones de seguidores refleja la decidida apuesta que el medio ha realizado por destacar en las redes sociales con una política muy activa de movilización y generación de contenidos, así como con el desarrollo de estrategias específicas dirigidas a conseguir seguidores y proyectar su visibilidad. Esta misma situación se observa en El País, con más de 2,6 millones de seguidores. En los dos casos, la intensidad de su actividad en la red es importante aunque no es el factor determinante, ya que El País supera los 110.000 tuits cuando 'Muy Interesante apenas supera los 30.000 y, aún así, su posicionamiento es mejor que el del periódico. A esta 
reflexión habría que sumar la constatación sobre la importancia de ofrecer contenidos especializados en la red con contenidos exclusivos capaces de llegar con información de muy alta calidad a un sector muy concreto de usuarios.

Aquí podríamos destacar la importancia de factores ya apuntados como la confianza y la credibilidad y recalcar que, pese al carácter de comunicación inmediata que supone Twitter, la calidad de los contenidos es un elemento discriminatorio y clave desde el punto de vista del posicionamiento. En el caso de Muy Interesante, la publicación que encabeza el ranking de medios, hay que sumar además su apuesta por contenidos curiosos relacionados con la ciencia, la historia, la tecnología, la naturaleza, la salud o la cultura con un enorme atractivo y que funcionan muy bien en las redes. Igual ocurre con el caso de Marie Claire, la revista francesa femenina que se ha abierto un importantísimo mercado en otros mercados como el español o el estadounidense con sus contenidos de moda, salud y belleza. Otra apuesta segura en las Red si se realiza una estrategia tan solvente como la que han llevado a cabo los responsables de las dos revistas.

Si nos fijamos en el ranking de medios (Tabla II), los dos principales diarios deportivos (Marca y $A s$ ) se encuentran en los primeros puestos (Marca, el tercero; As, el quinto). Sin embargo, su posición en la Figura 1 es bien diferente, ya que presentan patrones opuestos. En el caso del $A s$ su director, Alfredo Relaño, se comporta en cierto modo como Ramírez y logra posicionarse en cuarto lugar con más de los 100.000 seguidores y con una relación parecida a la del director de El Mundo (1:5). Por su parte, el comportamiento de Marca es inverso, ya que la cuenta del medio es mucho más poderosa que los de su director $(1: 45)$ y por tanto se suma a los medios ubicados cerca del eje horizontal. En este caso, debemos matizar que se trata del director de MarcaTV, Felipe del Campo, y no del máximo responsable del rotativo deportivo, Oscar Campillo, una excepcionalidad que es determinante en sus respectivas posiciones. Felipe del Campo ha logrado construirse una posición muy sólida dirigiendo en canal de televisión de Marca, como él mismo dice "la televisión del fútbol en abierto". Es evidentemente que Del Campo se beneficia de la marca del periódico pero hubiera sido interesante saber cómo se comportaría tanto Campillo con su perfil personal como en relación a Marca de haber apostado por Twitter como su equivalente $\operatorname{del} A s$.

En el duelo periodístico de información general y deportivos -que claramente ganan los deportivos en los quioscos-, lo que sí podemos observar es que en el escenario digital la competencia está más discutida. El País, con una potente estrategia impulsada por el Grupo Prisa, domina a gran distancia del resto y El Mundo se posiciona prácticamente en la misma escala que Marca (1,3 millones de seguidores) y, a algo más de distancia, As. Esta dura competencia se rompe, sin embargo, cuando hablamos de los directores, donde sale claramente beneficiado Pedro J. impulsado por su propio perfil y por el impacto que le reporta su medio. En todo caso, son dos ajenos a los medios tradicionales los que dominan el escenario, algo que debería tenerse muy en cuenta por parte de los directivos de los periódicos si realmente está en sus agendas de transformación y supervivencia utilizar las redes sociales como un ámbito más de oportunidad y negocio. 
Del mismo modo esto ocurre con los medios que se ocupan de forma específica por las noticias, por la actualidad más inmediata, -es el caso de EFE Noticias $\left(10^{\circ}\right.$ lugar $)$, Terra Noticias $\left(15^{\circ}\right)$ y 24 Horas Noticias $\left(18^{\circ}\right)$ - o por la economía (Expansión ocupa el $19^{\circ}$ puesto). Junto con los diarios de información general de referencia (El País, El Mundo, 20 Minutos, ABC, Público o La Vanguardia) también destacan algunos de los ciberdiarios más populares (El Confidencial y Diario.es) y, por supuesto, la ventana a las principales televisiones (RTVE, Telecinco o La Sexta) y a la radio líder en España (Cadena Ser). En concreto, RTVE ocupa una posición muy destacada, con la mitad de seguidores que El Mundo, y también es destacable la particular relación que se produce entre directivos de los dos diarios deportivos de mayor tirada en papel, muy en la línea de lo que hemos visto respecto a El Mundo y El País.

Llegados a este punto debemos destacar una de las primeras conclusiones que arroja el gráfico: el vacío que se observa en la parte superior derecha de la imagen; no hay ningún directivo de los medios mejor posicionados que logre un lugar de liderazgo. Si bien la mayoría de los directivos se acumulan junto con sus medios en la parte inferior izquierda denotando su escasa visibilidad e influencia en la red (aquí tendríamos a todos los medios regionales y locales, revistas, ciberdiarios, radios y televisiones mencionados al analizar la muestra), llama la atención, por ejemplo, observar cómo el director del periódico mejor posicionado (El País) ni siquiera sobresale en el gráfico. Javier Moreno se sitúa en la zona 'outsider' junto al resto de medios, un lugar que podríamos explicar tanto por la baja intensidad de su actividad en la red como por la poca proyección de su marca personal (la ratio en este caso llega a 1:78). A diferencia de El Mundo, un periódico muy personalista plenamente identificado con Pedro J. Ramírez, en el El País la figura del director está mucho más diluida. Ocurre igual con los directores de los dos periódicos deportivos, que también consiguen buenos posicionamientos impulsados por el efecto marca de sus medios. No obstante, en estos dos casos la intensidad de su actividad les ofrece una visibilidad importante en el gráfico que no logra alcanzar el director de El País.

$\mathrm{Si}$ volvemos al ranking de directores inicial, hay algunas otras observaciones que podemos realizar sobre el perfil de los profesionales que integran 'top 10' y el 'top 20'. En primer lugar, es destacable que sólo una mujer se 'cuela' en el top ten. Se trata de Montserrat Domínguez, con un ratio de 41.800, la periodista dio el salto desde uno de los programas más escuchados de la Ser (el matinal del fin de semana) a dirigir la edición española de The Huffington Post, el diario online más leído en Estados Unidos y uno de los medios online más innovadores en la red que tiene además como 'marca de la casa' situar a mujeres al frente de la dirección.

Otra interesante observación es el cruzado de datos entre el ranking de directores y de medios, ya que no se corresponde cien por cien. Es el caso, justamente, de El Huffington, ya que el medio en sí no aparece en el ranking pero sí dos personas vinculadas al diario: su directora y Gumersindo Lafuente. Sobre este último hay que matizar que estamos ante un caso similar a Ignacio Escolar, un periodista que se ha construido su posición y marca en la red al margen del medio al que ha estado vinculado en cada momento de su intensa etapa profesional. Durante los tres últimos años fue el responsable del cambio digital de El País, entre 2007 a 2009 estuvo al frente de Soitu.es 
como fundador y previamente fue la cabeza visible del proyecto digital de El Mundo (de 2000 a 2006).

En el ranking de directores se encuentran, asimismo, dos líderes de Eldiario.es (el director y el subdirector) e igual ocurre con 20 Minutos (aparece su director, Arsenio Escolar $^{3}$, padre de Ignacio Escolar, y la subdirectora, Virginia P. Alonso), al tiempo que hay directores cuyos medios ni siquiera aparecen en el ranking correspondiente: es el caso especial ya comentado de Julio Alonso, de Marhuenda con La Razón (el periódico como tal no logra situarse en los puestos más relevantes) y, sobre todo, de periodistas que se han convertido en verdaderos líderes de opinión en la Red con iniciativas exclusivamente como el diario Ara.cat, que fue en su día una apuesta fortísima por el mercado catalán (hablamos de Carles Capdevila), o al frente de blogs de enorme éxito como es el caso de Trecebits (sobre periodismo 2.0, tecnología e internet) y Clipset (sobre tecnología y gadgets con un marcado carácter noticioso). Tanto Capdevila como Manuel Moreno (Trecebits) y Carolina Denia (Clipset) están en el 'top 20'pero no sus medios de referencia.

Una caso remarcable es el de Carmela Ríos (Terra Noticias), una periodista analógica que trabajó en Cuatro, CNN+ y Antena 3 y que dio el salto al bando 3.0 con la cobertura del movimiento 15-M a través de Twitter y que logró por tal despliegue el Premio Ortega y Gasset al mejor trabajo en periodismo digital. En este caso, tanto la 'bloguera' como Terra aparecen entre los rankings de líderes en Twitter.

Y relevante es también la posición de Antonio Rodicio, que acaba de ser nombrado director de la Cadena Ser, y de Carlos Marañón, director de Cinemanía y articulista del diario As. Su presencia, además, podríamos explicarla con criterios similares: tanto Rodicio como Marañón se ha construido su posición con el paraguas y proyección de medios tan solventes como la Ser, Cinemanía y As. En el caso Marañón, hijo, nieto y sobrino de futbolistas, su usuario en Twitter no es su marca personal sino el binomio @ futbolycine uniendo dos puntales tan populares en la redes como el cine y el fútbol.

Por último, si nos centramos en el listado de medios, los casos más llamativos de medios de enorme tirón que no tienen correspondencia con los profesionales que los dirigen son los mencionados Muy Interesante y Marie Claire en el ámbito de las revistas especializadas, el periódico $A B C$ en tanto que su director Bieito Rubido apenas cuentan con 10.000 seguidores cuando el diario supera los $345.000 \mathrm{o}$ el caso de las televisiones y medios audiovisuales, donde los periodistas o comunicadores que realmente tienen impacto en Twitter no son los directores del medio sino perfiles marcadamente mediáticos que, como dijimos anteriormente, quedan fuera del listado por la distorsión que implicaría para el enfoque periodístico de la muestra con la que estamos trabajando.

${ }^{3}$ Es director del diario gratuito líder en España desde su fundación y presidente de la Asociación Española de Editoriales de Publicaciones Periódicas (AEEPP). Antes ha trabajado también como subdirector de El País, subdirector de Cinco Días, director del diario Claro, redactor jefe del diario El Sol y director de Diario 16 de Burgos. 


\section{Conclusiones}

Podemos concluir que, si bien la marca y liderazgo del medio en el mercado tradicional es un factor clave para impulsar la posición del director en las redes sociales, no es el único requisito. La marca contribuye pero han de darse otros condicionantes que podríamos sintetizar en la idea de 'marca personal' que estamos defendiendo.

Si tomamos como referencia el liderazgo de los periódicos en cuando a audiencia y difusión que se produce en el mercado tradicional, también podríamos llamar la atención sobre el hecho de que sean los dos grandes diarios nacionales de información general y los dos deportivos de mayor tirada los que alcancen una posición influyente en la red. Resulta llamativo comprobar que otros diarios nacionales de gran proyección como $A B C$ no aparezcan destacados, como también ocurre con La Razón o La Gaceta. Los regionales tampoco sobresalen (contrasta por ejemplo la escasa visibilidad de los diarios del grupo Vocento a pesar de la fuerte apuesta por internet que ha realizado el grupo y los positivos datos que arrojan sus webs) e igual ocurre con diarios de gran tradición e influencia en el panorama periodístico como los catalanes $L a$ Vanguardia y El Periódico.

Yendo concretamente a la primera pregunta planteada al principio del trabajo, podemos recalcar cómo las cuentas de Twitter de los medios son, en la inmensa mayoría, mucho más importantes que la de sus propios directores. El caso paradigmático en este sentido es el de El País.

Con relación a la segunda pregunta, solo se puede contestar afirmativamente en muy pocos casos. El más destacado es el de Ignacio Escolar, quién dobla en número de seguidores a su propio medio. El caso de Alonso es en los números aún más acentuado, aunque en este caso no estamos ante un medio tradicional en el estricto sentido de la palabra. Si bien puede haber algún otro caso, se trata de cantidades tan pequeñas que son despreciables y que no pueden tenerse en cuenta. Podemos decir, por tanto, que estamos ante solo cuatro casos de personas que se constituyen en marcas por sí solos y que podríamos definir como brand-directors: Ignacio Escolar, Pedro J. Ramírez, Alfredo Relaño y Julio Alonso.

Con relación a la tercera pregunta, hay que destacar que la ratio directores/medios es en términos globales de 1 en 10. En el caso peculiar de los brand-directors tenemos tasas positivas de 8:1 (Alonso), 2:1 (Escolar) o ligeramente negativas de 1:6 (Ramírez) y 1:5 (Relaño), pero en la inmensa mayoría los medios son muy superiores en seguidores. Por su parte, para directivos de medios muy populares presentan tasas bajas tales como 1:45 (Marca), 1:78 (El País), 1:180 (RTVE), e incluso hasta 1:900 (Muy Interesante). En todos estos casos los directores no han querido o sabido utilizar el nombre del medio para potenciar su canal personal.

Por último, debemos indicar que este se trata de un primer estudio de aproximación a la problemática sobre el uso de la herramienta Twitter en el periodismo español. Seguimos trabajando en el líneas que nos ha abierto este primer trabajo. Entre ellas se destaca el análisis dinámico de este colectivo, donde pueda apreciarse la evolución que puede tener cada uno de estos directores y medios a lo largo de todo un año. Otro elemento que será analizado son las ausencias. Este trabajo habla de las principales presencias en Twitter, pero no hemos profundizado en las ausencias, que son 
también llamativas, o en los casos de presencia pero muy baja actividad. Finalmente, otro punto de análisis interesante será el análisis de contenido de estas cuentas, con el fin de poder caracterizar la actividad de un director frente a los contenidos propios de un medio. Este último análisis podría darnos algunas claves que expliquen los comportamientos descritos en el presente trabajo.

\section{Referencias bibliográficas}

CARRERA ÁlVAREZ, Pilar; SAINZ DE BARANDA ANDÚJAR, Clara; HERRERO CURIEL, Eva et al. (2012): "Journalism and Social Media: How Spanish Journalists are Using Twitter". Estudios sobre el Mensaje Periodístico, vol. 18, $\mathrm{n}^{\circ}$ 1, pp. 31-53. Madrid, Servicio de Publicaciones de la Editorial Complutense. [doi:10.5209/rev_eSMP.2012.v18.n1.39353]

EDO BOLÓS, Concepción (2002): Del papel a la pantalla. La prensa en Internet. Sevilla, Comunicación Social Ediciones y Publicaciones.

ELÓSEGUI, Tristán (2011): "Los medios usan Twitter como un altavoz". Analitica Web: http://tristanelosegui.com/2011/01/23/los-medios-de-comunicacion-usantwitter-como-un-altavoz/ [fecha de consulta: 08 de octubre de 2013].

HERRERO-SOLANA, Víctor y HASSAN-MONTERO, Yusef (2005): "Metodologías para el desarrollo de interfaces visuales de recuperación de información: análisis y comparación". Information Research, vol 11, n 3 , Lund, Lund University Libraries, paper 258. http://informationr.net/ir/11-3/paper258.html [fecha de consulta: 08 de octubre de 2013].

HERRERO-SOLANA, Víctor y TRILLO-DOMÍNGUEZ, Magdalena (2013). “Information about Twitter account of spanish journalists and mass media". ZENODO, Ginebra, CERN, doi:10.5281/zenodo.6957. http://zenodo.org/record/6957 [fecha de consulta: 08 de octubre de 2013].

MARK DE ZABALETA (2011) "Twitter ya cuenta con casi todos los directores de periódicos españoles". 233 Grados: http://233grados.lainformacion.com/blog /2011/06/directores-medios-twitterhtml[fecha de consulta: 08 de octubre de 2013].

NOBIL AHMAD, Ali (2010): "Is Twitter useful tool for journalists?". Journal of Media Practice, vol. 11, $\mathrm{n}^{\mathrm{o}}$ 2, London, Intellect Ltd., pp. 145-155. [doi:10.1386/jmpr.11.2.145_1]

ORIHUELA, José Luis (2012): 80 claves sobre el futuro del periodismo. Madrid, Anaya.

RODRÍGUEZ RUIBAL, Antonio y GARCÍA LÓPEZ, Javier (2013): "Uso y funciones de Twitter en periodistas españoles". Estudios sobre el Mensaje Periodístico, Vol. 19, Núm. especial abril, pp. 963-969. Madrid, Servicio de Publicaciones de la Editorial Complutense. [doi:10.5209/rev_ESMP.2013.v19.42181]

SALAVERRÍA, Ramón (2007): "Los diarios frente al reto digital”, en Revista Latinoamericana de Comunicación Chasqui, nº 97, pp. 4-9, Quito, CIESPAL. 
TRILLO-DOMÍNGUEZ, Magdalena (2008): Análisis cibermétrico de la prensa digital española: ranking de calidad web y mapa de influencia mediática. Tesis doctoral de la Universidad de Granada, Departamento de Biblioteconomía y Documentación.

TRILLO-DOMÍNGUEZ, Magdalena y MOYA-ANEGÓN, Félix (2008): “Aproximación cienciométrica a la investigación en comunicación: el caso de Marshall McLuhan". El Profesional de la Información, vol. 17, $\mathrm{n}^{\circ}$ 3. Barcelona, Metapress, pp. 303-309. [doi:10.3145/epi.2008.may.06]. 